\title{
CHARACTERIZATION OF SOLID STATE ARRAY CAMERAS FOR THE MID-IR
}

\author{
Eric Keto, ${ }^{1,2}$ Roger Ball, ${ }^{2,3}$ John Arens, ${ }^{2,4}$ \\ Garrett Jernigan, ${ }^{4}$ Margaret Meixner ${ }^{2,5}$ \\ ${ }^{1}$ University of California \\ Lawrence Livermore National Laboratory \\ POB 808 L-59 \\ Livermore, California 94550
}

Received May 23, 1992

\begin{abstract}
We present a characterization of some processes affecting the performance of solid state array cameras designed for ground based astronomical imaging in the $8-13 \mu \mathrm{m}$ atmospheric window. Our discussion includes a novel model for electron-hole generationrecombination noise based on the probable pathlength of an electron in a photoconductor. We use the Berkeley mid-IR Array Camera as an example. For this camera, the results show that the total optical system composed of the camera, a $3 \mathrm{~m}$ telescope, and the atmosphere has an efficiency of about $3 \%$, a $1 \sigma$ noise equivalent flux density of $25 \mathrm{mJy} \min ^{-1 / 2} \operatorname{arcsec}^{-2}$ measured over a $\Delta \lambda / \lambda=10 \%$ band width, and a noise equivalent expressed as the ambient temperature thermal black body noise of $23 \%$.

subject headings: Infrared Astronomy; Infrared Instruments; Infrared Imaging; Detector Arrays

${ }^{2}$ Visiting Astronomer, NASA Infrared Telescope Facility, University of Hawaii; ${ }^{3}$ Participating Guest, Institute of Geophysics and Planetary Physics, Lawrence Livermore National Laboratory; ${ }^{4}$ Space Sciences Laboratory, University of California, Berkeley; ${ }^{5}$ Department of Astronomy, University of California, Berkeley
\end{abstract}




\section{Introduction}

While solid state array detectors are commonly used in optical and near-IR astronomy, the high mid-IR background $(8-13 \mu \mathrm{m}$ atmospheric window) has made operation of solid state array detectors quite difficult in this spectral region. However, employing newer generations of detectors with deeper well depths and higher speed signal processors, there are now several mid-IR array cameras in operation or under construction $[1,2,3,4,5]$. In this paper we describe some processes affecting the performance of solid state array cameras operating in the mid-IR. As part of the discussion, we characterize the performance of one of the first cameras, the Berkeley mid-IR array camera. After a brief description of the instrument, we discuss factors affecting the efficiency and noise, and means by which the performance may be characterized.

\section{The Berkeley mid-IR Array Camera}

The Berkeley mid-IR array camera was designed and developed at the Space Sciences Laboratory, University of California, Berkeley. The camera is operated jointly by SSL and the Institute of Geophysics and Planetary Physics at the Lawrence Livermore National Laboratory. The detector is a Hughes Aircraft $10 \times 64$ liquid helium cooled, gallium doped silicon photoconductor array. The electronics are designed for rapid reading of the photoconductor array for use in the presence of the high background rates typical of the $8-13 \mu \mathrm{m}$ spectral region at ground based sites. The photoconductor array has 10 read out lines allowing parallel addressing of the individual pixel columns and a read rate of $20 \mu \mathrm{sec}$ per pixel. The camera may be configured for a frame rate of $780 \mathrm{~Hz}$ using all 64 pixels per column or a higher frame rate for a subset of the 64 pixels. The camera can be operated with an $8-13 \mu \mathrm{m}$ N-band filter or with $1 \%$ or $10 \%$ circular variable filters (CVF). The N-band transmissivity is close to the transmissivity of the curve labeled $N^{\prime}$ in figure 1 . The imaging optics allow for a range of plate scales typically from 0.3 to $1.0^{\prime \prime}$. The camera design itself is described in a previously published paper [6]. More recent advances in architecture, which are incorporated in a new camera currently under construction for the Keck Observatory are described in [7].

The camera is typically operated with alternate observations 


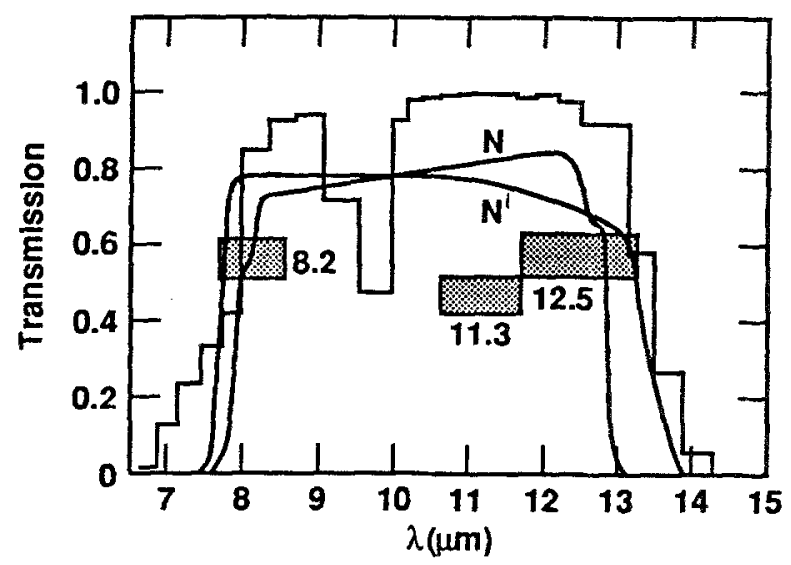

Figure 1. Square blocked line, transmission of an ideal atmosphere [8]. Shaded boxes show the location of the pass bands used in our experiments with respect to the transmission of the atmosphere. Lines labeled $\mathrm{N}$ and $\mathrm{N}^{\prime}$ show the transmission of two filters used with the IRTF bolometer for comparison. 
of an astronomical source and blank sky with offsets produced by moving the secondary telescope mirror (chop) at a rate set by the number of frames co-added at each sky position and by moving the telescope itself (nod) at a slower rate set by the number of on-off chop pairs. The observations reported here were made with 10000 co-added frames resulting in an integration time of $12.8 \mathrm{~s}$ at each position in the nod-chop cycle. The data discussed in this paper were taken with the camera mounted on the NASA Infrared Telescope Facility (IRTF).

\section{Efficiency}

The performance of an astronomical instrument is coupled with the performance of the entire optical path along which information travels from the astronomical source to the observer. In figure 2, we list for reference the information flow from incident photons at the top of the atmosphere to analog to digital converter (ADC) counts. As is typical for a ground based mid-IR system, the major contributions to noise in the system derive from the atmosphere and the telescope. In comparison, the contributions to the noise deriving from dark current and chip read noise are both negligible.

We may consider three measures of the efficiency: the efficiency of the total system composed of the atmosphere, telescope, and camera; the efficiency of the detector in converting photons to current; and the quantum efficiency of the photoconductor. The ratio of the last two efficiencies is the photoconductive gain which is the efficiency in converting photoelectrons in the photoconductor to current or electrons collected on the anode of the photoconductor. The photoconductive gain is adjustable, depending primarily on the bias voltage applied across the detector, but the performance of individual photoconductor chips is difficult to predict. The following simple model for photoelectron generation and hole recombination suggests a means of measuring the photoconductive gain.

\subsection{Photoconductive Gain}

A photoelectron released in the detector and traveling toward the anode under the influence of the bias voltage applied across the detector has a probability of recombining with a hole. If the 


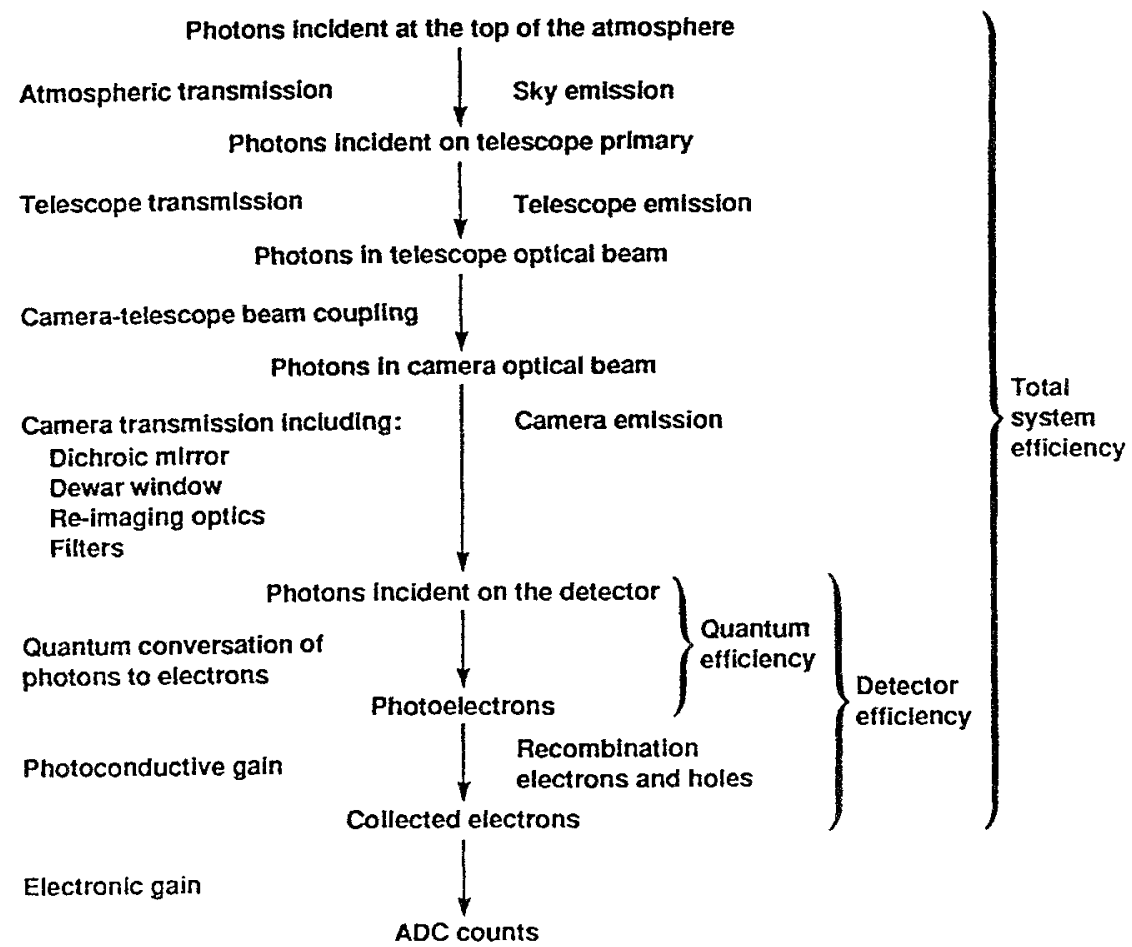

Figure 2. Summary of the processes affecting the quality of ground based mid-IR images. The left column shows the different transmissions and conversions in the optical path, the middle column defines position of the photons or electrons, and the right column shows the sources of emission. The annotations on the far right define the conversion efficiencies referred to in the text. See text §IIIa for a more complete explanation. 
probability of recombination is constant along the path then,

$$
P(s)=\frac{1}{\lambda} e^{-s / \lambda}
$$

The mean path length and standard deviation are

$$
\begin{gathered}
\bar{s}=\int_{0}^{\infty} P(s) s d s=\frac{1}{\lambda} \int_{0}^{\infty} s e^{-s / \lambda} d s=\lambda \\
\sigma=\left(\int_{0}^{\infty} P(s)(s-\bar{s})^{2} d s\right)^{1 / 2}=\lambda
\end{gathered}
$$

For conditions of high count rates, the voltage signal or number of electrons collected on the anode can be expressed as the total length of the paths of $n$ photoelectrons.

$$
n_{c}=\frac{S}{h}
$$

where the total path is the sum of all the individual paths

$$
S=\sum_{i=1}^{n} s_{i}
$$

and $S>>h$, where $h$ is the thickness of the detector.

In this case the noise deriving from the uncertainty due to recombination may be seen as equivalent to the uncertainty in the path length of the $n$ photoelectrons, while the noise deriving from fluctuations in the incoming emission will be equivalent to the uncertainty in the number $n$ of photoelectrons. The two uncertainties combine in the total uncertainty of the outgoing signal. The uncertainty in the number of photoelectrons is straightforwardly the square root of the number of photoelectrons. We now determine the uncertainty in the pathlength of $n$ photoelectrons. The probability that two electrons will have a combined path of length exactly equal to $S$ is

$$
P_{2}=\int_{0}^{S} P(s) P(S-s) d s
$$


This is equivalent to a convolution of the pathlength probability distribution of a single electron with itself. We may express the convolution as a product of the single-sided Laplace transforms of the functions

$$
\begin{gathered}
L\left[P_{2}(S)\right]=L[P(S)] L[P(S)] \\
P_{2}(S)=L^{-1}\left[(L[P(S)])^{2}\right]
\end{gathered}
$$

and by extension

$$
P_{n}(S)=L^{-1}\left[(L[P(S)])^{n}\right]
$$

The Laplace transform of an exponential has a simple form

$$
L\left[e^{-S / \lambda}\right]=\frac{1}{t-1 / \lambda}
$$

and therefore

$$
\left(L\left[\frac{1}{\lambda} e^{-S / \lambda}\right]\right)^{n}=\frac{1}{\lambda^{n}} \frac{1}{(t-1 / \lambda)^{n}}
$$

and

$$
P_{n}(S)=\frac{1}{(n-1) !}\left(\frac{S}{\lambda}\right)^{n-1} \frac{1}{\lambda} e^{-S / \lambda}
$$

Note that this probability distribution of $n$ electrons traveling a total length $S$ is different from the joint probablility of $n$ electrons each individually traveling a length $S / n,(P(S / n))^{n}=\frac{1}{\lambda^{n}} e^{-S / \lambda}$.

We may now compute the mean path length in the distribution $P_{n}$ and the standard deviation using equations 2 and 3. The mean path length is

$$
\bar{S}=\int_{0}^{\infty} \frac{1}{\lambda^{n}} \frac{1}{(n-1) !} S^{n} e^{-S / \lambda} d S
$$

This equation may be integrated by parts. The first integration yields

$$
\bar{S}=\frac{1}{\lambda^{n}} \frac{1}{(n-1) !}\left(\left[-\lambda S^{n} e^{-S / \lambda}\right]_{0}^{\infty}-n \lambda \int_{0}^{\infty} S^{n-1} e^{-S / \lambda} d S\right)
$$


Thus each of $n$ integrations by parts contributes a factor of $\lambda$ and factors of $n,(n-1),(n-2) \ldots$ respectively. The final integration of $S^{0} e^{-S / \lambda}$ contributes an additional factor of $\lambda$. The result is that

$$
\bar{S}=n \lambda
$$

By a similar analysis, the standard deviation (equation 3 ) comes out to

$$
\sigma_{S}=\sqrt{n} \lambda
$$

The signal is $n_{c}=\frac{S}{h}=\frac{n \lambda}{h}$ and the total uncertainty is

$$
\begin{gathered}
\sigma_{n c}=\left(\sigma_{S}^{2}\left(\frac{\partial n_{c}}{\partial S}\right)^{2}+\sigma_{n}^{2}\left(\frac{\partial n_{c}}{\partial n}\right)^{2}\right)^{1 / 2} \\
\sigma_{n c}=\left((\sqrt{n} \lambda)^{2}\left(\frac{1}{h}\right)^{2}+(\sqrt{n})^{2}\left(\frac{\lambda}{h}\right)^{2}\right)^{1 / 2} \\
\sigma_{n c}=\sqrt{2 n} \frac{\lambda}{h}
\end{gathered}
$$

The signal to noise ratio is

$$
r=\sqrt{n / 2}
$$

Thus the signal to noise ratio is independent of the photoconductive gain which is

$$
g_{p c}=\frac{n_{c}}{n}=\frac{\lambda}{h}
$$

Therefore the noise caused by fluctuations in the photon flux and by variations in path length are unaffected by photoconductive gain. If there are no variations in path length, the signal to noise ratio is $\sqrt{N}$. This case occurs in an impurity blocked detector where the path lengths are identically equal to the detector thickness and the photoconductive gain is therefore unity.

It might seem that the factor of $\sqrt{2}$ could be derived by assuming that the statistical fluctuations in the generation of photoelectrons contribute a term of $\sqrt{N}$ to the noise while the fluctuations in the recombination of photoelectrons contribute an additional term of $\sqrt{N}$. Added in quadrature these two terms 
would imply the correct level of noise $\sqrt{2 N}$; however, this cannot be right. This would assume that the recombination noise depends only on the mean number of photoelectrons $N$, but is independent of the actual number of photoelectrons in the conductor, which is on the order of $N \pm \sqrt{N}$ at any particular time. This is clearly not the case; for example, at high counts, the fluctuations in recombination model, could exceed the total number of free electrons in the photoconductor.

Our model suggests a means of calculating the photoconductive gain. Since the photoconductive gain is similar to the gain of the back end electronics in that $N$ and $\sqrt{N}$ are changed by identical factors - except for the factor of $\sqrt{2}-$ the gains in converting from ADC counts to photoelectrons cancel out in the ratio of measured ADC counts to the measured rms fluctuations in counts. We may therefore compute the number of photoelectrons in the photoconductor as $N_{p e}=2\left(\left(N-N_{b}\right) / \Delta N\right)^{2}$ where $N_{b}$ is the count rate due to any applied bias voltage and the factor of 2 reflects the recombination noise. For example, using the Berkeley mid-IR Array Camera, observation of an approximate black body at $299 \mathrm{~K}$ with a $0.16 \%$ band pass centered at $12.8 \mu \mathrm{m}$ yields a lower limit to the photoelectron rate of $10^{8}$ $\mathrm{s}^{-1}$. We determine the photoconductive gain from the counts per photoelectron accounting for the known gains of the back end electronics, $g_{p c}=\left(\right.$ counts $\left./ N_{p e}\right) /($ back end gain $)=0.27$.

\subsection{Detector, Quantum and Total Efficiencies}

Given the photoelectron rate, we may estimate the quantum efficiency of the detector from the rate of incoming photons. The number of photons emitted by a $299 \mathrm{~K}$ blackbody is of course $B_{\nu} A \Omega \Delta \nu T$, the Planck function times the detector area, (accounting for any magnification in the optics), the beam size, the band width, and the transmission of the optics. For example, in the Berkeley camera, the individual pixels have a width of $120 \mu \mathrm{m}$, the re-imaging optics in the camera are set to create an $\mathrm{f} / 35$ light cone and a demagnification of 1.7 , while the optics themselves have a net transmission of 0.52 . Thus in an observation of a black body, the number of photons striking a pixel is $1.5 \times 10^{9} \mathrm{~s}^{-1}$ over a band width of $0.16 \mu \mathrm{m}$ implying a lower limit to the quantum efficiency, $q . e .=$ photons $/$ photoelectrons $=7 \%$. 
The quantum efficiency of our Hughes Aircraft photoconductor array ought to be about $10 \%$. Our measurements are consistent with this estimate. In comparison, the newer RIBIT or BIB chips should achieve $25 \%$ while a single pixel bolometer achieves about $80 \%$.

Both the derived quantum efficiency and the photoconductive gain represent lower limits because noise which may arise from causes other than the statistical $\sqrt{N}$ noise will reduce the estimate of the number of photoelectrons by an amount proportional to the inverse square of the magnitude of the additional fluctuations. For example, the temperature of the black body may change with air currents in the lab, and the electronics may also add noise to the derived counts. A calculation of the system efficiency in the next section suggests that a photoelectron rate $30 \%$ higher than the lower limit derived above may be more appropriate. Such a rate would be achieved if $15 \%$ of the fluctuation amplitude were induced by other causes. Fluctuations of the temperature of the black body of a few milli-Kelvin would be sufficient.

Using the derived photoconductive gain along with the known electronics gain, we may compute the total system efficiency, defined here as the ratio of photons above the atmosphere to photoelectrons, from observations of calibrator stars of known flux density. Table 1 lists the relevant data. The derived system efficiency is $4.3 \%$. We may ask what is the expected efficiency. The atmospheric transmission is 0.9 , using a model atmosphere [8]; the telescope transmission is difficult to measure, but is expected to be about $85 \%$; the camera optics have a transmission of 0.46 ; the quantum efficiency is $\geq 0.07$; and in addition we measure an optical coupling between the telescope and camera beams of 0.8 (see $§ 4$ ); The expected efficiency is therefore $2.5 \%$. If as mentioned before, the number of photoelectrons were higher by $15 \%$, our derived photoconductive gain would be lower by $30 \%$, the quantum efficiency higher by $30 \%$, and both the expected and observed total system efficiencies would be about $3 \%$.

\section{Effective Emissivity}

We estimate the effective emissivity of the atmospheretelescope-instrument system by comparing the noise power of 
Table 1

Measurements of Calibrator Stars

\begin{tabular}{llll}
\hline \hline Star & $\begin{array}{l}\text { wavelength* } \\
(\mu \mathrm{m})\end{array}$ & $\begin{array}{l}\text { Flux } \\
(\mathrm{Jy})\end{array}$ & photoelectrons s \\
\hline & & & \\
$\gamma$ Aql & 8.2 & 116 & $5.3 \times 10^{7}$ \\
$\alpha$ Cyg & 8.2 & 31.5 & $1.1 \times 10^{7}$ \\
$\alpha$ Tau & 8.2 & 926 & $3.4 \times 10^{8}$ \\
$\gamma$ Aql & 8.2 & 116 & $4.5 \times 10^{7}$ \\
$\beta$ Peg & 11.3 & 335 & $1.4 \times 10^{8}$ \\
$\gamma$ Aql & 12.5 & 55 & $3.9 \times 10^{7}$ \\
$\alpha$ Tau & 12.5 & 426 & $2.0 \times 10^{8}$ \\
$\alpha$ Her & 12.5 & 994 & $3.9 \times 10^{8}$ \\
$\alpha$ Tau & $\mathrm{N}$ & 648 & $2.6 \times 10^{8}$ \\
$\beta$ And & $\mathrm{N}$ & 260 & $1.1 \times 10^{8}$ \\
\hline \hline
\end{tabular}

* Measurements at $8.2,11.3$, and $12.5 \mu \mathrm{m}$ made with a $10 \%$ circular variable filter. Measurements at N-band are centered approximately at $10.6 \mu \mathrm{m}$ with a $5 \mu \mathrm{m}$ band width. 
the system (blank sky) with the noise power of a black body represented by the closed cavity of the telescope structure and mirror cover. We obtain an effective emissivity of $23 \%$ from the ratio of counts in an observation made with the telescope mirror cover open (blank sky) and closed (black body). The uncertainty is on the order of a few percent arising from atmospheric fluctuations.

Using the same considerations, we may measure the coupling between the upward looking camera beam and the downward looking telescope beam. The re-imaging optics of the camera and the alignment procedure seek to exactly illuminate the entire primary mirror. To investigate the coupling, we make measurements with the mirror cover closed, and one with the mirror cover closed except for a small hatch in the center of the mirror cover (figure 3 ). The open hatch allows the small diameter upward looking camera beam to see the secondary, but the larger downward looking beam reflected off the secondary sees the top of the mirror cover and not the primary mirror. If the coupling is perfect, the two measurements will give the same power. If the camera beam is too large and escapes around the secondary, then the hatch open reading will be lower than the hatch closed reading. If the camera beam is too small, the measurements will yield the same power and this error cannot be determined from this experiment. The coupling determined this way is $80 \%$. The expected error in the coupling measurement again derives primarily from the variation in the atmospheric emissivity and is on the order of a few percent.

We may ask what is the expected effective emissivity. Starting with an hypothetical black body above the atmosphere, we may move the black body to the position of the mirror covers by correcting for the transmission and emission of the atmosphere and telescope. We may then determine the effective emissivity by comparing a measurement of blank sky to this hypothetical observation as a ratio. The number of predicted photoelectrons and the number of electrons measured in an observation of blank sky are listed in table 2. The effective emissivity calculated this way is within the expected errors identical to that calculated in the mirror cover experiment.

\section{Noise Equivalent Flux Density}

To determine the expected noise level of an astronomical 

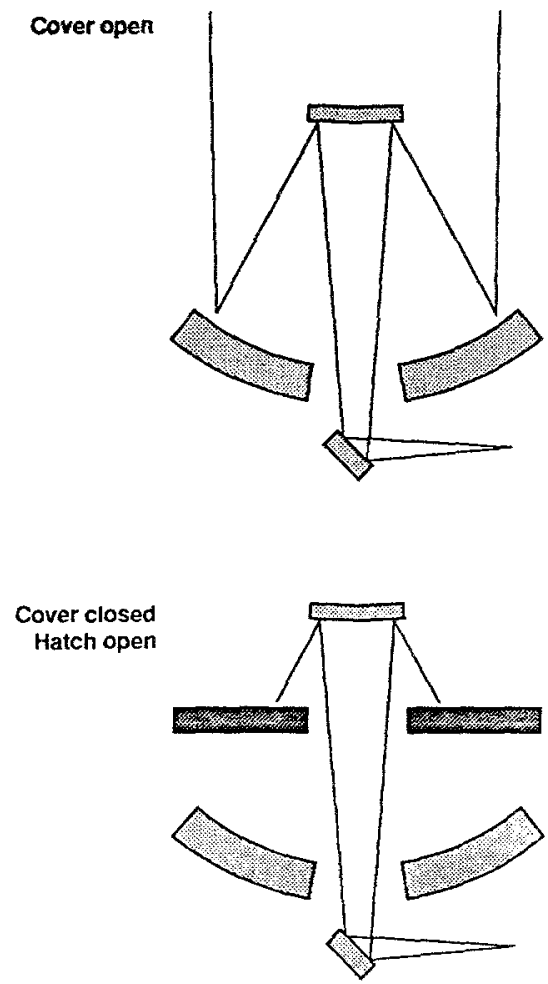

Cover closed
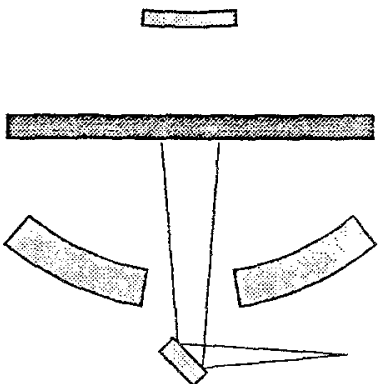

Figure 3. Schematic for the beam coupling experiments described in the text. The diagrams depict the primary and secondary mirrors of the telescope and the $45^{\circ}$ dichroic mirror of the camera. The three diagrams show the ray paths for different configurations of the telescope mirror covers and the mirror cover hatch. 
Table 2

Effective Emissivity

\begin{tabular}{llll}
\hline \hline wavelength* & $\begin{array}{l}\text { Black Body } \\
\text { photons } \\
\left(\mathrm{s}^{-1}\right)\end{array}$ & $\begin{array}{l}\text { Black Body } \\
\text { photoelectrons } \\
\left(\mathrm{s}^{-1}\right)\end{array}$ & $\begin{array}{l}\text { effective } \\
\text { emmisivity }\end{array}$ \\
\hline $8.2^{* *}$ & $3.4 \times 10^{10}$ & $2.9 \times 10^{9}$ & .41 \\
12.5 & $8.3 \times 10^{10}$ & $6.5 \times 10^{9}$ & .28 \\
\hline
\end{tabular}

* Measurements at 8.2 , and $12.5 \mu \mathrm{m}$ made with a $10 \%$ circular variable filter.

** The effective emissivity is high at $8.2 \mu \mathrm{m}$ because part of the band width extends beyond the atmospheric window (see figure 2). 
observation, we determine $\Delta N$ as $\sqrt{2 N}$ where $\mathrm{N}$ is the number of photoelectrons in the observations of blank sky from Table 2. The noise level may be converted from photoelectrons $\mathrm{s}^{-1}$ pixel-1 to $\mathrm{Jy} \mathrm{s}^{-1} \operatorname{arcsec}^{-2}$ using the calibrator measurements in Table 1 . The estimated NEFD is about $15 \mathrm{mJy} \mathrm{min}-1 / 2 \operatorname{arcsec}^{-2}$ with a $10 \%$ band pass at $12.5 \mu \mathrm{m}$.

We may compare this noise estimate with the noise measured in a calibrated sky map. Maps made with a $10 \%$ band width have noise levels of about $25 \mathrm{mJy} \mathrm{min} \mathrm{m}^{-1 / 2} \operatorname{arcsec}^{-2}$ [9]. The noise level in an actual observation is higher than that estimated as $\Delta N=\sqrt{N}$ because the noise in the map includes additional sources of noise owing primarily to fluctuations in the sky brightness over time. Figure 4 is a plot of photoelectrons over time. The variation is primarily due to fluctuations in sky brightness caused by passing cirrus clouds. The periodic cycling is caused by the chopping of the secondary mirror.

What is the actual background noise expected for a perfect efficiency detector operating under an ideal sky [8] model atmosphere, with no haze (particles) and no cirrus? From Traub and Stier, the sky centered on $10 \mu \mathrm{m}$ in a $10 \%$ passband has a brightness of 0.515 Watts $\mu \mathrm{m}^{-1}$ steradian ${ }^{-1} \mathrm{~m}^{-2}$. This is equivalent to a flux of $4.3 \times 10^{9}$ photons s$^{-1}$ arcsec $^{-2}$. The telescope, a $280 \mathrm{~K}$ black body with an emissivity of 0.15 , produces a flux of $8.7 \times 10^{9}$ photons $\mathrm{s}^{-1} \operatorname{arcsec}^{-2}$. Multiplying the sky flux by the telescope transmission of 0.85 and adding the telescope flux presents a total background flux of $1.23 \times 10^{10}$ photons $\mathrm{s}^{-1} \operatorname{arcsec}^{-2}$ to the hypothetical $10 \mu \mathrm{m}$ instrument. The statistical fluctuations will be $\sqrt{N}=1.1 \times 10^{5}$ photons $\mathrm{s}^{-1} \operatorname{arcsec}^{-2}$, or $1.35 \mathrm{mJy} \mathrm{min}-1 / 2 \operatorname{arcsec}^{-2}$.

\section{Summary}

1. We present methods for calculating certain characteristics of solid state array cameras including the photoconductive gain.

2. The Berkeley mid-IR Array Camera has a total system efficiency including atmosphere of about $3 \%$, a detector efficiency of about $5 \%$, and an effective emissivity of the combined camera, telescope, and atmosphere of $30 \%$. The camera noise level for a $1 \mu \mathrm{m}$ band width is $25 \mathrm{mJy}$ $\min ^{-1 / 2} \operatorname{arcsec}^{-2}$.

Acknowledgements: Work performed under the auspices of 


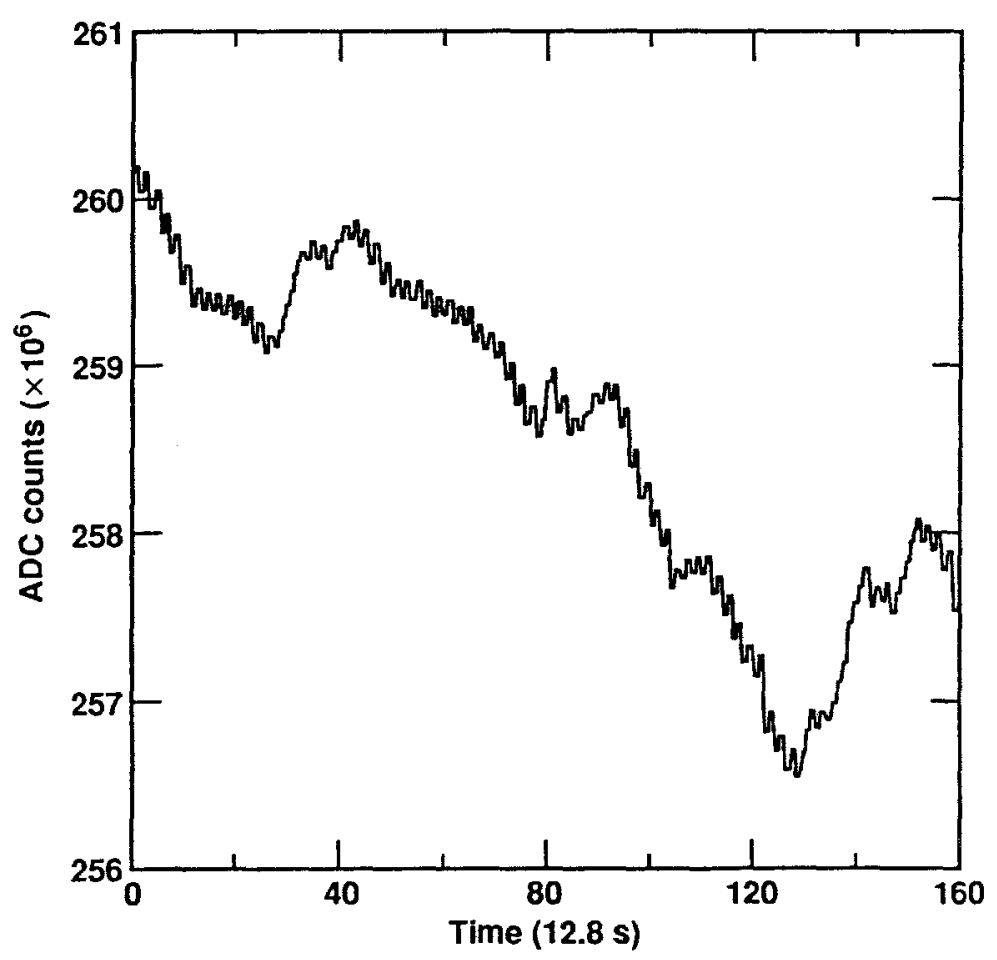

Figure 4. Measured analog to digital converter counts for an observation at $12.5 \mu \mathrm{m}$. Each measurement represents 12.8 seconds of integration time. 
United States Department of Energy at Lawrence Livermore National Laboratory under contract number W-7405-ENG-48.

The NASA Infrared Telescope Facility is operated by the University of Hawaii under contract with the National Aeronautics and Space Administration.

\section{References}

[1] Keto, E.R., Ball, R., Arens, J., Jernigan, G., and Meixner, M. 1991, Ap. J. Lett., 374, L29

[2] McCaughrean, M. and Gezari, D. 1991, in Astrophysics with Infrared Arrays, ed. R. Elston, PASP, San Francisco

[3] Hoffmann, W., Hora, J., Fazio, G., Shivananden, K. 1991, Mirac Report No 10., University of Arizona, Tucson, AZ

[3] Hora, J. 1991, PhD Thesis, University of Arizona, Tucson, AZ

[4] Puetter, R., Jones, B., and Pina, R., 1990, Final Report:NAG 2-580 'Low Background Array Testing'

[5] Odenwald, S., Shivananden, K., and Thronson, H. 1991, preprint

[6]Arens, J.F., Jernigan, J.F., Peck, M.C., Lacy, J., \& Gaalema, S. 1987, Applied Optics, 26, 3846

[7]Peck, M.C., Arens, J.F., Jernigan, J.G., \& Gaalema, S. 1988, November, IEEE Transactions on Nuclear Science, Orlando, FL

[8] Traub, W., \& Stier, M. 1976, Applied Optics, 15, 364

[9]Keto, E., Ball, R., Arens, J.F., Jernigan, G., \& Meixner, M. 1990, Ap. J., in press 